\title{
Searching for New Physics in the rare decay $B^{+} \rightarrow D_{s}^{+} \phi$
}

\author{
Rukmani Mohanta \\ School of Physics, University of Hyderabad, Hyderabad-500 046, India
}

\begin{abstract}
The rare decay $B^{+} \rightarrow D_{s}^{+} \phi$ can occur only via annihilation type diagram in the standard model. The small branching ratio predicted in the standard model makes this channel sensitive to new physics contributions. We analyze this decay mode, both in the standard model and in several extensions of it. The models considered are minimal supersymmetric model with R-parity violation and two Higgs doublet model. The experimental verification of our findings of large branching ratio and/or nonzero CP asymmetry may signal the presence of new physics.
\end{abstract}

Typeset using REVTEX 
Recent results from the ongoing experiments in $B$-physics at BABAR and BELLE have attracted a lot of attention. The main objective of these $B$ experiments is to explore in detail the physics of $\mathrm{CP}$ violation, to determine many of the flavor parameters of the standard model (SM) at high precision and to probe for possible effects of new physics beyond the SM [1 [3]. The intensive search for Physics beyond the standard model is performed now a days in various areas of particle physics. The $B$ system thus offers a complementary probe to the search for new physics. In $B$ experiments, new physics beyond the SM may manifest itself in the following two ways :

$i$. decays which are expected to be rare in the SM and are found to have large branching ratios.

ii. CP violating asymmetries which are expected to vanish or to be very small in the SM are found to be significantly large.

Thus the rare $B$ meson decays are suggested to give good opportunities for discovering new physics beyond the SM. Since their branching ratios are small in the SM, they are very sensitive to new physics contributions. In the last few years, different experimental groups have been accumulating plenty of data for the rare $B$ decay modes. Some of them have already been measured by the $B$ factories in KEK and SLAC.

In this context it is interesting to analyze the rare decay $B^{+} \rightarrow D_{s}^{+} \phi$, which is a pure annihilation type decay in the SM. The four valence quarks in the final states $D_{s}$ and $\phi$ are different from the ones in the parent $B$ meson, i.e. there is no spectator quark in this decay. In the usual factorization approach, this decay mode can be described as the $\bar{b}$ and $u$ quarks in the initial $B$ meson annihilating into vacuum and the final $D_{s}$ and $\phi$ mesons are produced from the vacuum afterwards. The dynamics of exclusive hadronic $B$ decays occurring via the $W$ - exchange or annihilation diagrams, is not yet understood. The decay rates for such transitions are argued to be negligibly small due to the suppression of helicity and (or) form factors. However, a solid justification of this argument is necessary in both theory and experiments. This decay mode has been recently studied in perturbative QCD approach with a branching ratio $3 \times 10^{-7}$, 4 , which is far below the current experimental 
upper limit [5]

$$
\operatorname{Br}\left(B^{+} \rightarrow D_{s}^{+} \phi\right)<3.2 \times 10^{-4}
$$

Therefore it provides an appropriate testing ground for physics beyond the SM.

In this paper we intend to study the decay mode $B^{+} \rightarrow D_{s}^{+} \phi$, both in the standard model and in several extensions of it. The models considered here are the Two Higgs Doublet Model (2HDM) [6] and minimal supersymmetric model with R-parity violation (RPV) [7].

We first consider the contributions arising from the SM, where the effective Hamiltonian describing the decay mode is given as

$$
\mathcal{H}_{e f f}=\frac{G_{F}}{\sqrt{2}} V_{u b}^{*} V_{c s}\left[C_{1}(\mu) O_{1}(\mu)+C_{2}(\mu) O_{2}(\mu)\right]
$$

where

$$
\begin{aligned}
& O_{1}=\left[\bar{b} \gamma_{\mu}\left(1-\gamma_{5}\right) u\right]\left[\bar{c} \gamma^{\mu}\left(1-\gamma_{5}\right) s\right] \\
& O_{2}=\left[\bar{b} \gamma_{\mu}\left(1-\gamma_{5}\right) s\right]\left[\bar{c} \gamma^{\mu}\left(1-\gamma_{5}\right) u\right]
\end{aligned}
$$

and $C_{1,2}(\mu)$ are Wilson coefficients evaluated at the renormalization scale $\mu$. In the next to leading logarithmic approximation their values are evaluated at the $b$ quark mass scale as 8

$$
C_{1}\left(m_{b}\right)=1.082 \quad \text { and } \quad C_{2}\left(m_{b}\right)=-0.185
$$

The corresponding transition amplitude is given as

$$
\operatorname{Amp}\left(B^{+} \rightarrow D_{s} \phi\right)=\frac{G_{F}}{\sqrt{2}} V_{u b}^{*} V_{c s} a_{1}\left\langle D_{s}^{+} \phi\left|\left(\bar{c} \gamma^{\mu}\left(1-\gamma_{5}\right) s\right)\left(\bar{b} \gamma_{\mu}\left(1-\gamma_{5}\right) u\right)\right| B^{+}\right\rangle
$$

where $a_{1}=C_{1}+C_{2} / N_{c}$, with $N_{C}$ is the number of colors. The evaluation of the matrix element of four fermion operator from the first principles of QCD is an extremely demanding challenge. To have some idea of the magnitudes of matrix element, one can still use the factorization method, factorizing the four quark operators relevant to non leptonic $B$ decays into the product of two currents and evaluating separately the matrix elements of the two 
currents. Thus in the factorization approximation one can write the corresponding transition amplitude as

$$
\operatorname{Amp}\left(B^{+} \rightarrow D_{s}^{+} \phi\right)=\frac{G_{F}}{\sqrt{2}} V_{u b}^{*} V_{c s} a_{1}\left\langle D_{s}^{+} \phi\left|\left(\bar{c} \gamma^{\mu}\left(1-\gamma_{5}\right) s\right)\right| 0\right\rangle\left\langle 0\left|\left(\bar{b} \gamma_{\mu}\left(1-\gamma_{5}\right) u\right)\right| B^{+}\right\rangle
$$

The main uncertainties in evaluating the transition amplitude are due to the matrix element like $\left\langle D_{s}^{+} \phi\left|\left(\bar{c} \gamma^{\mu}\left(1-\gamma_{5}\right) s\right)\right| 0\right\rangle$ with $\left(p_{D_{s}}+p_{\phi}\right)^{2} \simeq M_{B}^{2}$. To get an idea about the contributions from annihilation diagrams, one can assume single pole dominance for the matrix element and relate it to the crossed channel $\left\langle\phi\left|\bar{s} \gamma^{\mu}\left(1-\gamma_{5}\right) c\right| D_{s}\right\rangle$. We see that the annihilations do not give a large contributions here : with single pole dominance, form factors are suppressed by a factor $M_{D_{s}}^{p 2} /\left(M_{B}^{2}-M_{D_{s}}^{p 2}\right)$ because the transferred momentum $\sqrt{Q^{2}}=M_{B}$ is large with respect to pole mass $M_{D_{S}}^{p}$. The annihilation contribution could be larger if there were other pseudoscalar $(c \bar{s})$ resonators heavier than the pole mass $M_{D_{s}}^{p}$, which would enhance the form factors. The matrix element of the pseudoscalar and vector meson is usually decomposed as [9]

$$
\begin{aligned}
\langle\phi(q, \epsilon)| \bar{s} \gamma^{\mu}(1 & \left.-\gamma_{5}\right) c\left|D_{s}(p)\right\rangle=\frac{2 V\left(Q^{2}\right)}{M_{D_{s}}+M_{\phi}} \epsilon^{\mu \nu \alpha \beta} \epsilon_{\nu}^{*} p_{\alpha} q_{\beta} \\
& -i(\epsilon \cdot Q) \frac{2 M_{\phi}}{Q^{2}} Q^{\mu}\left(A_{3}\left(Q^{2}\right)-A_{0}\left(Q^{2}\right)\right) \\
& +i\left(M_{D_{s}}+M_{\phi}\right)\left[\epsilon^{\mu *} A_{1}\left(Q^{2}\right)-\frac{\epsilon^{*} \cdot Q}{\left(M_{D_{s}}+M_{\phi}\right)^{2}}(p+q)^{\mu} A_{2}\left(Q^{2}\right)\right]
\end{aligned}
$$

where $Q=(p-q)$ is the momentum transferred during the transition process. Using the decay constant relation

$$
\left\langle 0\left|\bar{b} \gamma^{\mu} \gamma_{5} u\right| B^{+}(Q)\right\rangle=i f_{B} Q^{\mu}
$$

the transition amplitude in the $\mathrm{SM}$ is given as

$$
\operatorname{Amp}\left(B \rightarrow D_{s} \phi\right)=-\frac{G_{F}}{\sqrt{2}} V_{u b}^{*} V_{c s} a_{1} f_{B} 2 M_{\phi} A_{0}\left(M_{B}^{2}\right)\left(\epsilon^{*} \cdot Q\right)
$$

and the corresponding decay width as

$$
\Gamma\left(B \rightarrow D_{s} \phi\right)=\frac{p_{c}^{3}}{8 \pi M_{\phi}^{2}}\left|\operatorname{Amp}\left(B \rightarrow D_{s} \phi\right) /\left(\epsilon^{*} \cdot Q\right)\right|^{2},
$$


where $p_{c}$ is the c.m. momentum of the decay particles. The value of the form factor $A_{0}(0)$ at zero momentum transfer for the transition $D_{s} \rightarrow \phi$ is given as $9 A_{0}(0)=0.7$ and the $Q^{2}$ dependence of the form factor is given as

$$
A_{0}\left(Q^{2}\right)=\frac{1}{1-\frac{Q^{2}}{M_{P}^{2}}}
$$

where $M_{P}$ is the pole mass with value $M_{P}=1.97 \mathrm{GeV}$ [9]. Thus we get

$$
A_{0}\left(M_{B}^{2}\right)=-0.113
$$

Using $a_{1}=1.04$ [10], which is extracted from the experimental data on $B \rightarrow D^{*}(\pi, \rho), f_{B}=190$ $\mathrm{MeV}$, the CKM matrix elements from [5] and $\tau_{B}=1.653 \times 10^{-12} s$, we obtain the branching ratio in the $\mathrm{SM}$ as

$$
\operatorname{Br}\left(B^{+} \rightarrow D_{s}^{+} \phi\right)=1.88 \times 10^{-6}
$$

Thus one can see that the obtained branching ratio in the SM using the factorization assumption is far below the experimental value $\operatorname{Br}\left(B^{+} \rightarrow D_{s}^{+} \phi\right)<3.2 \times 10^{-4}$. Furthermore, it should be noted here that the direct CP asymmetry for this decay mode is zero in the SM since it receives contribution only from the single annihilation diagram.

We now proceed to evaluate the branching ratio in the QCD Improved factorization method, which has been developed recently [11] to study the hadronic $B$ decays. This method incorporates elements of naive factorization approach as its leading term and perturbative QCD corrections as subleading contributions and thus allowing one to compute systematic radiative corrections to the naive factorization for hadronic $B$ decays. This method is expected to give a good estimate of the magnitudes of the hadronic matrix elements in non leptonic $B$ decays. However, in the QCD factorization approach the weak annihilation contributions are power suppressed as $\Lambda_{Q C D} / m_{b}$ and hence do not appear in the factorization formula. Besides power suppression they also exhibit end point singularities even at twist two order in the light cone expansion of the final state mesons and therefore can not be computed self consistently in the context of hard scattering approach. One possible 
way to go around the problem is to treat the end point divergence arising from different sources as different phenomenological parameters [12]. The corresponding price one has to pay is the introduction of model dependence and extra numerical uncertainties. In this work we will follow the treatment of [12 and express the weak annihilation amplitude for $B^{+} \rightarrow D_{s}^{+} \phi$ as

$$
\operatorname{Amp}\left(B^{+} \rightarrow D_{s}^{+} \phi\right)=\frac{G_{F}}{\sqrt{2}} V_{u b}^{*} V_{c s} f_{B} f_{D_{s}} f_{\phi} b_{1}\left(D_{s}, \phi\right)
$$

The annihilation parameter $b_{1}\left(D_{s}, \phi\right)$ is given as

$$
b_{1}\left(D_{s}, \phi\right)=\frac{C_{F}}{N_{c}} C_{1} A_{1}^{i}\left(D_{s}, \phi\right)
$$

where the color factor $C_{F}=\frac{N_{C}^{2}-1}{2 N_{C}}$ and $N_{C}=3$. The function $A_{1}^{i}\left(D_{s}, \phi\right)$ is given as

$$
A_{1}^{i}\left(D_{s}, \phi\right)=\pi \alpha_{s} \int_{0}^{1} d x \int_{0}^{1} d y \psi_{D_{s}}(x) \psi_{\phi}(y)\left[\frac{1}{y(1-x \bar{y})}+\frac{1}{\bar{x}^{2} y}\right]
$$

where $\psi_{D_{s}}(x)$ and $\psi_{\phi}(y)$ are the light cone distribution amplitudes (LCDA) for the final mesons and $x$ is the longitudinal momentum fraction of $c$ quark in $D_{s}$ and $\bar{y}$ is the momentum fraction of $\bar{s}$ in $\phi$. Using the assumption that the LCDAs of the mesons $D_{s}$ and $\phi$ are symmetric, one can parameterize the weak annihilation contribution as [13]

$$
A_{1}^{i}\left(D_{s}, \phi\right) \simeq 18 \pi \alpha_{s}\left(X_{A}+\frac{\pi^{2}}{3}-4\right)
$$

where $X_{A}=\int_{0}^{1} d x / x$ parameterizes the end point divergence as

$$
X_{A}=\int_{0}^{1} \frac{d x}{x}=\ln \frac{M_{B}}{\bar{\Lambda}}+\rho e^{-i \theta}
$$

$\rho$ varies from 0 to 6 and $\theta$ is an arbitrary phase $0<\theta<360^{\circ}$. Using $\bar{\Lambda}=\Lambda_{Q C D}=200$ $\mathrm{MeV}$ and $\rho e^{i \theta}=i \pi$ as default values along with $f_{B}=190 \mathrm{MeV}, f_{\phi}=233 \mathrm{MeV}, f_{D_{s}}=280$ $\mathrm{MeV}, C_{1}\left(m_{b}\right)=1.082$ and $\alpha_{s}\left(m_{b}\right)=0.221$, we obtain the branching ratio in QCD improved factorization approach as

$$
\operatorname{Br}\left(B^{+} \rightarrow D_{s}^{+} \phi\right)=0.67 \times 10^{-6}
$$


We now proceed to calculate the branching ratio for this decay mode in type II of Two Higgs Doublet Model [6]. In type II 2HDM, the up-type quarks get mass from one doublet, while down-type quarks and charged leptons from the other doublet. Charged Higgs Yukawa couplings are controlled by the parameter $\tan \beta=v_{2} / v_{1}$, the ratio of vacuum expectation values of the two doublets, normally expected to be of order $m_{t} / m_{b}$. For our concern the $H^{ \pm}$effectively induce the four fermion interaction as

$$
\begin{aligned}
\mathcal{H}_{e f f}^{2 H D M} & =-\frac{G_{F}}{\sqrt{2}} \frac{V_{u b}^{*} V_{c s}}{m_{H}^{2}}\left\{\bar{u}\left[m_{b} X\left(1+\gamma_{5}\right)+m_{u} Y\left(1-\gamma_{5}\right)\right]\right\} \\
& \times\left\{\bar{s}\left[m_{c} Y\left(1+\gamma_{5}\right)+m_{s} X\left(1-\gamma_{5}\right)\right] c\right\},
\end{aligned}
$$

where $m_{H}$ denotes the mass of the lightest charged scalar particle, $m_{q}$ 's denote the constituent quark masses and $X \simeq 1 / Y=v_{2} / v_{1}=\tan \beta$. In the above equation the terms proportional to $Y$ can be safely neglected as $X$ is generally taken as large [14]. To evaluate the matrix elements we use the equation of motion to transform the $(S-P)(S+P)$ currents to corresponding $(V-A)(V+A)$ form as

$$
\bar{q}_{1}\left(1 \pm \gamma_{5}\right) q_{2}=i\left[\frac{\partial^{\mu}\left(\bar{q}_{1} \gamma_{\mu} q_{2}\right)}{m_{q_{2}}-m_{q_{1}}} \mp \frac{\partial^{\mu}\left(\bar{q}_{1} \gamma_{\mu} \gamma_{5} q_{2}\right)}{m_{q_{2}}+m_{q_{1}}}\right]
$$

where the quark masses are current quark masses. Thus we obtain the expressions for matrix elements as

$$
\left\langle 0\left|\left(\bar{b} \gamma_{5} u\right)\right| B^{+}\right\rangle=-\frac{i f_{B} M_{B}^{2}}{m_{u}+m_{b}}
$$

and

$$
\left\langle\phi\left|\bar{s} \gamma_{5} c\right| D_{s}\right\rangle=i(\epsilon \cdot Q) \frac{2 M_{\phi}}{m_{s}+m_{c}} A_{0}\left(Q^{2}\right)
$$

Using the above relations, one can obtain the transition amplitude in 2HDM as

$$
\left.\operatorname{Amp}\left(B^{+} \rightarrow D_{s} \phi\right)\right|_{2 H D M}=\frac{G_{F}}{\sqrt{2}} V_{u b}^{*} V_{c s} \frac{\tan ^{2} \beta}{M_{H}^{2}} f_{B} \frac{m_{b} m_{s} M_{B}^{2}}{\left(m_{b}+m_{u}\right)\left(m_{s}+m_{c}\right)}(\epsilon \cdot Q) 2 M_{\phi} A_{0}\left(Q^{2}\right)
$$

So the total amplitude in 2HDM including SM contributions

$$
\mathcal{A}^{S M+2 H D M}=\mathcal{A}^{S M}\left(1-R_{1}\right)
$$


where

$$
R_{1}=\frac{1}{a_{1}} \frac{\tan ^{2} \beta}{M_{H}^{2}} \frac{m_{b} m_{s} M_{B}^{2}}{\left(m_{b}+m_{u}\right)\left(m_{s}+m_{c}\right)}
$$

The free parameters of the $2 \mathrm{HDM}$ namely $\tan \beta$, and $M_{H}$ are not arbitrary, but there are some semi quantitative restrictions on them using the existing experimental data. The most direct bound on charged Higgs boson mass comes from the top quark decays, which yield the bound $M_{H}>147 \mathrm{GeV}$ for large $\tan \beta[15]$. Furthermore, there are no experimental upper bounds on the mass of the charged Higgs boson, but one generally expects to have $M_{H}<1 \mathrm{TeV}$ in order that perturbation theory remains valid [16]. For large $\tan \beta$ the most stringent constraint on $\tan \beta$ and $M_{H}$ is actually on their ratio, $\tan \beta / M_{H}$. The current limits come from the measured branching ratio for the inclusive decay $B \rightarrow X \tau \bar{\nu}$, giving $\tan \beta / M_{H}<0.46 \mathrm{GeV}^{-1}$ [14. Using the constituent quark masses as $m_{b}=4.88 \mathrm{GeV}$, $m_{s}=0.5 \mathrm{GeV}$ [17] and current quark masses at the $b$ quark mass scale as $m_{b}=4.34 \mathrm{GeV}$, $m_{c}=0.95 \mathrm{GeV}, m_{s}=90 \mathrm{MeV}$ and $m_{u}=3.2 \mathrm{MeV}$ [18, the branching ratio in 2HDM is found to be

$$
\left.\operatorname{Br}\left(B^{+} \rightarrow D_{s}^{+} \phi\right)\right|_{2 H D M}=8.0 \times 10^{-6}
$$

We now analyze the possibility of observing direct $\mathrm{CP}$ violation in this decay mode since it now receives contributions both from the SM and 2HDM. We can write the decay amplitude (25) as

$$
\mathcal{A}^{S M+2 H D M}=\left|\mathcal{A}^{S M}\right| e^{i\left(\gamma+\delta_{1}\right)}\left[1-\left|R_{1}\right| e^{i(\phi+\delta)}\right]
$$

where $\gamma=\arg \left(V_{u b}^{*}\right)$ and $\delta_{1}$ are the weak and strong phases of standard model amplitudes. $\phi$ and $\delta$ are the relative weak and strong phases between 2HDM and SM amplitudes. Thus the direct CP asymmetry for the decay mode is given as

$$
\begin{aligned}
a_{c p} & =\frac{\operatorname{Br}\left(B^{+} \rightarrow D_{s}^{+} \phi\right)-B r\left(B^{-} \rightarrow D_{s}^{-} \phi\right)}{\operatorname{Br}\left(B^{+} \rightarrow D_{s}^{+} \phi\right)+B r\left(B^{-} \rightarrow D_{s}^{-} \phi\right)} \\
& =\frac{2\left|R_{1}\right| \sin \phi \sin \delta}{1+\left|R_{1}\right|^{2}-2\left|R_{1}\right| \cos \phi \cos \delta}
\end{aligned}
$$


If we set $\phi=\delta=\pi / 2$, the maximum possible value of direct CP violation in $2 \mathrm{HDM}$ is found to be

$$
\left.a_{c p}\left(B^{+} \rightarrow D_{s}^{+} \phi\right)\right|_{2 H D M} \leq 59 \%
$$

We now analyze the decay mode in minimal supersymmetric model with R-parity violation. In the supersymmetric models there may be interactions which violate the baryon number $B$ and the lepton number $L$ generically. The simultaneous presence of both $L$ and $B$ number violating operators induce rapid proton decay which may contradict strict experimental bound. In order to keep the proton lifetime within experimental limit, one needs to impose additional symmetry beyond the SM gauge symmetry to force the unwanted baryon and lepton number violating interactions to vanish. In most cases this has been done by imposing a discrete symmetry called R-parity defined as $R_{p}=(-1)^{(3 B+L+2 S)}$, where $S$ is the intrinsic spin of the particles. Thus the $R$-parity can be used to distinguish the particle $\left(R_{p}=+1\right)$ from its superpartner $\left(R_{p}=-1\right)$. This symmetry not only forbids rapid proton decay, it also render stable the lightest supersymmetric particle (LSP). However, this symmetry is ad hoc in nature. There is no theoretical arguments in support of this discrete symmetry. Hence it is interesting to see the phenomenological consequences of the breaking of R-parity in such a way that either $B$ and $L$ number is violated, both are not simultaneously violated, thus avoiding rapid proton decays. Extensive studies has been done to look for the direct as well as indirect evidence of R-parity violation from different processes and to put constraints on various $\mathrm{R}$-parity violating couplings. The most general $R$-parity and Lepton number violating super-potential is given as

$$
W_{L /}=\frac{1}{2} \lambda_{i j k} L_{i} L_{j} E_{k}^{c}+\lambda_{i j k}^{\prime} L_{i} Q_{j} D_{k}^{c},
$$

where, $i, j, k$ are generation indices, $L_{i}$ and $Q_{j}$ are $S U(2)$ doublet lepton and quark superfields and $E_{k}^{c}, D_{k}^{c}$ are lepton and down type quark singlet superfields. Further, $\lambda_{i j k}$ is antisymmetric under the interchange of the first two generation indices. Thus the relevant four fermion interaction induced by the R-parity and lepton number violating model is 


$$
\mathcal{H}_{R,}=-\frac{\lambda_{2 i 2}^{\prime} \lambda_{i 13}^{\prime *}}{4 M_{\tilde{e}_{L_{i}}}^{2}} \bar{u}\left(1-\gamma_{5}\right) b \bar{s}\left(1+\gamma_{5}\right) c
$$

where the summation over $i=1,2,3$ is implied. It should be noted that the $R P V$ Hamiltonian has the same form as the 2HDM Hamiltonian except the couplings. So using the Eqs. (22) and (23) one can easily obtain the amplitude for the $B^{+} \rightarrow D_{s}^{+} \phi$ in $R P V$ model,

$$
\left.\operatorname{Amp}\left(B^{+} \rightarrow D_{s}^{+} \phi\right)\right|_{R P V}=\frac{\lambda_{2 i 2}^{\prime} \lambda_{i 13}^{*}}{4 M_{\tilde{e}_{L_{i}}}^{2}} \frac{f_{B} M_{B}^{2}}{\left(m_{b}+m_{u}\right)\left(m_{s}+m_{c}\right)}(\epsilon \cdot Q) 2 M_{\phi} A_{0}\left(Q^{2}\right)
$$

and the total amplitude as

$$
\mathcal{A}^{S M+R P V}=\mathcal{A}^{S M}\left(1-R_{2}\right)
$$

where

$$
R_{2}=\frac{\sqrt{2}}{G_{F}} \frac{1}{V_{u b}^{*} V_{c s} a_{1}} \frac{\lambda_{2 i 2}^{\prime} \lambda_{i 13}^{\prime *}}{4 M_{\tilde{e}_{L_{i}}}^{2}} \frac{M_{B}^{2}}{\left(m_{b}+m_{u}\right)\left(m_{s}+m_{c}\right)}
$$

Using $\lambda_{2 i 2}^{\prime} \lambda_{i 13}^{* *}=2.88 \times 10^{-3}$ [19], we obtain the branching ratio in RPV model as

$$
\operatorname{Br}\left(B^{+} \rightarrow D_{s}^{+} \phi\right)=3.06 \times 10^{-4}
$$

Thus we found that the branching ratio in RPV model is quite large in comparison to SM prediction. So if the experimental value is found to be in this range, it will definitely be a signal of new physics beyond the SM.

Replacing $R_{2}$ in place of $R_{1}$ in Eq. (29), the direct CP asymmetry in the decay mode $B^{+} \rightarrow D_{s}^{+} \phi$ in RPV model is found to be

$$
\left.a_{c p}\left(B^{+} \rightarrow D_{s}^{+} \phi\right)\right|_{R P V} \leq 14 \%
$$

To conclude, in this paper we have calculated the branching ratio of the two body hadronic decay mode $B^{+} \rightarrow D_{s}^{+} \phi$ in the standard model as well as in the two Higgs doublet model and RPV model. We found that the branching ratio in the RPV model is quite large in comparison to the SM prediction, whereas the $2 \mathrm{HDM}$ prediction is approximately one order higher than the SM value. The direct $\mathrm{CP}$ asymmetry $a_{c p}$, which is expected to be zero in the SM, is found to be nonzero and large in both RPV and 2HDM analyses. From our analyses it follows that the new physics contribution is quite large and significant. Therefore the rare decay mode $B^{+} \rightarrow D_{s}^{+} \phi$ provides an ideal testing ground to look for new physics. 


\section{REFERENCES}

[1] P. F. Harrison and H. R. Quinn, Editors The Babar Physics Book, SLAC-R-504 (1998).

[2] M. T. Cheng et al, Belle Collaboration, KEK Report 94-2; F. Takasaki hep-ex/9912004.

[3] R. Fleischer and J. Matias, Phys. Rev. D 61, 074004 (2000).

[4] C.-D. Lü, hep-ph/0112127.

[5] D. E. Groom et al, Review of Particle Physics, Euro. Phys. J. C 15, 1 (2000).

[6] S. Glashow and S. Weinberg, Phys. Rev. D 15, 1958 (1977).

[7] C. S. Aulakh and R. N. Mohapatra, Phys. Lett. B 119, 136 (1982); F. Zwirner, Phys. Lett. 132, 103 (1983); I.-H. Lee, Nucl. Phys. B 246, 120 (1984); J. Ellis et al, Phys. Lett. B 150, 142 (1985); G. G. Ross and J. W. F. Valle, Phys. Lett. 151, 375 (1985); S. Dawson, Nucl. Phys. B 261, 295 (1985); R. Barbieri, A. Masiero, Nucl. Phys. B 267, 679 (1986): S. Dimopoulos and L. Hall, Phys. Lett. B 207, 210 (1987).

[8] G. Buchalla, A. J. Buras and M. E. Lautenbacher, Rev. Mod. Phys. 68, 1125 (1996).

[9] M. Bauer, B. Stech and M. Wirbel, Z. Phys. 34, 103 (1987).

[10] H.-Y. Cheng and K.-C. Yang, Phys. Rev. D 59, 092004 (1999).

[11] M. Beneke, G. Buchalla, M. Neubert and C. T. Sachrajda, Phys. Rev. Lett. 83, 1914 (1999); Nucl. Phys. B 591, 313 (2000).

[12] M. Beneke, G. Buchalla, M. Neubert and C. T. Sachrajda, Nucl. Phys. B 606, 245 (2000).

[13] D. Du, H. Gong, J. Sun, D. Yang and G. Zhu, Phys. Rev. D 65, 094025 (2002).

[14] ALEPH Collaboration in ICHEP'96, Proceedings of the 28th International Conference on High Energy Physics, Warsaw, Poland edit. Z. Ajdeck and A. Wroblewski, World Scientific, Singapore (1997). 
[15] F. Abe et. al., CDF Collaboration, Phys. Rev. Lett. 79, 357 (1997).

[16] M. Veltman, Phys. Lett. B 70, 253 (1997).

[17] A. Ali, G. Kramer and C.-D. Lü, Phys. Rev. D 58, 094009 (1998).

[18] Y.-H. Chen, H.-Y. Cheng, B. Tseng and K. C. Yang, Phys. Rev. D 60, 094014 (1999).

[19] D. K. Ghosh, X.-G. He, B. H. J. McKellar and J.-Q. Shi, hep-ph/0111106. 